\title{
Meta-analysis of competitive antagonists in asthma treatment
}

\author{
Zhen Lei ${ }^{1}$, Qi Luo ${ }^{2}$, Weiyi Yang ${ }^{1}$, Qiaofeng Su ${ }^{1}$, Ying Zuo ${ }^{3}$ \\ ${ }^{1}$ Department of Respiratory and Critical Care Medicine, Affiliated Hospital of North Sichuan Medical College, Nanchong, China; ${ }^{2}$ Outpatient \\ Department, Affiliated Hospital of North Sichuan Medical College, Nanchong, China; ${ }^{3}$ Department of Endocrinology and Metabolism, Affiliated \\ Hospital of North Sichuan Medical College, Nanchong, China \\ Contributions: (I) Conception and design: Z Lei, Y Zuo; (II) Administrative support: W Yang; (III) Provision of study materials or patients: Z Lei, \\ Q Luo, Q Su, Y Zuo; (IV) Collection and assembly of data: All authors; (V) Data analysis and interpretation: Z Lei, Q Luo, W Yang, Q Su; (VI) \\ Manuscript writing: All authors; (VII) Final approval of manuscript: All authors. \\ Correspondence to: Ying Zuo. Department of Endocrinology and Metabolism, Affiliated Hospital of North Sichuan Medical College, No. 1 Maoyuan \\ South Road, Shunqing District, Nanchong, China. Email: yingzuo76@gmail.com.
}

\begin{abstract}
Background: This meta-analysis was developed to explore the efficacy of using antagonists to the chemokine receptor of T helper type 2 (Th2) cells (CRTH2) in the treatment of asthma, so as to provide theoretical foundation and assistance for treatment of asthma.

Methods: The Boolean logic search method (Boolean method) was adopted to search the publications in PubMed, Medline, and HowNet with "antagonist", "asthma”, "competitive antagonist", and "efficiency" as the search terms. The publications taking placebo as control to treat the asthma were searched. The Review Manager software was applied for meta-analysis.

Results: A total of 13 publications were included in this study, and most of them were low-risk bias (medium-high quality). The results of Meta-analysis showed that the treatment methods of the two groups of patients showed no statistically heterogeneous effects on the one-second forced expiratory volume (FEV1) in the maximum exhalation after the maximum deep inhalation $\left(\mathrm{Chi}^{2}=4.70, \mathrm{I}^{2}=0 \%\right.$, and $\left.\mathrm{P}=0.79\right)$. The FEV1 of the control group was much lower than that of the experimental group $(Z=3.61, P=0.0003)$. There was no great difference in the incidence of adverse events between the two groups $(Z=0.46, P=0.64)$. No statistical heterogeneity $\left(\mathrm{Chi}^{2}=3.72, \mathrm{I}^{2}=0 \%, \mathrm{P}=0.45\right)$ and difference $(\mathrm{Z}=1.57, \mathrm{P}=0.12)$ were found in asthma exacerbation between the two groups. The asthma control questionnaire (ACQ) score of the control group was much lower than that of the experimental group $(\mathrm{Z}=3.20, \mathrm{P}=0.001)$.
\end{abstract}

Conclusions: Competitive antagonists of CRTH2 could effectively improve FEV1 and lower ACQ scores in patients with asthma, and were therefore effective in treating asthma.

Keywords: Chemokine receptor; competitive antagonists; placebo; asthma exacerbation

Submitted Jan 13, 2021. Accepted for publication Mar 23, 2021.

doi: 10.21037/apm-21-412

View this article at: http://dx.doi.org/10.21037/apm-21-412

\section{Introduction}

Asthma is a respiratory disease characterized by chronic inflammation in the airways, which is affected by eosinophils, mast cells, lymphocytes, neutrophils, airway epithelial cells, and their components (1). Asthma can cause narrowing of the airways and affect the gas exchange in the lungs, which can lead to breathing difficulties. Asthma symptoms can suddenly deteriorate and patients can present with wheezing, coughing, chest tightness, abnormal breathing (rapid shallow breathing or dyspnea), sleep disorders, and fatigue (2,3). Severe asthma can cause a series of complications, such as pneumothorax (abnormal accumulation of gas in the pleural cavity), mediastinal emphysema, and atelectasis (obstructed abdominal expansion). In addition, long-term asthma or persistent infections can cause chronic obstructive pulmonary disease and pulmonary heart disease $(4,5)$. Patients show 
different degrees and types of the above symptoms due to the severity of asthma and different physical conditions $(6,7)$. Related surveys have found that there are currently more than 200 million people suffering from asthma in the world, of which 29 million are from China (8). However, the mechanisms and etiology of asthma has not yet been fully elucidated. It is generally believed that the pathogenesis of asthma is complicated and mostly related to genetic and environmental factors. People with a family history of asthma are more likely to develop asthma (9). In addition, exposure to allergens (dust, pollen, harmful gases, air pollution, and certain objects or drugs) in the living environment may cause the body's immune system to release inflammatory factors, thereby causing allergic reactions and asthma. Studies have found that women, after the age of 40 , are more likely to develop asthma than men of the same age (10).

The high-level expression of CRTH2 can increase the number of white blood cells (WBCs), cause destructive damage to the tissues, and result in some diseases. In addition, chemokines and their receptors can regulate the infiltration and activation of allergic inflammatory cells centered on eosinophils from the blood to the bronchial mucosa and are closely related to the pathophysiological process of asthma. Antagonists of Th2 cell chemokine receptors can be used as new targets for asthma treatment. The treatment of asthma is mainly to take medication and control possible triggers (that is, to avoid the patient's contact with irritating substances that may cause allergic reactions). Currently commonly used drugs for the treatment of asthma include inhaled corticosteroids, oral or injected corticosteroids (methylprednisolone, prednisone), short-acting $\beta 2$ receptor agonists (e.g., salbutamol), and some long-term drugs (anticholinergics, leukotriene modulators, and long-acting $\beta 2$ receptor agonists) (11). The drug exerts anti-inflammatory effects and anti-allergic reactions, helps patients expand their airways, relieve asthma symptoms, improve asthma control, and reduce their acute attacks. Competitive antagonists can compete with the agonist for the same receptor. By increasing the dose of agonist and antagonist to compete for the binding site, it can effectively prevent and reduce mucosal inflammatory cell infiltration, improve the patient's lung function, and reduce the number of lymphocytes and macrophages (12). Common competitive antagonist drugs include montelukast, zafirlukast, and pranlukast. Although existing studies have found that competitive antagonists can effectively prevent the onset of symptoms in children and adults with asthma, the sample size of such studies is generally small, and the research indicators are different, the research conclusions are biased, and there is a lack of competitive systematic review and evaluation of the effectiveness of the drug in the treatment of asthma. Therefore, a Meta-analysis method was applied in this study to explore the effectiveness of competitive antagonists in the treatment of asthma.

We present the following article in accordance with the PRISMA reporting checklist (available at http://dx.doi. org/10.21037/apm-21-412).

\section{Methods}

\section{Document retrieval}

Relevant literature was selected by the Boolean method, using "antagonist", "asthma”, "competitive antagonist", and "efficiency" as search terms in PubMed, Medline, EMbase, China Biomedical Literature Database, HowNet Database, Wanfang Database, Weipu Database, and Google Scholar. The time range of searched documents was from the establishment of the database to October 30, 2020. The quality of the documents was evaluated with the RevMan 5.2 software provided by the Cochrane system. The search terms were combined freely to search multiple times to confirm the selected literature. In addition, the latest research progress was obtained after consultation with experts and researchers in the field.

\section{Inclusion and exclusion criteria of the literature}

Studies included in this meta-analysis met all the following criteria: the report discussed the effectiveness of competitive antagonists in the treatment of asthma; the patients in the control group received conventional placebo treatment; the patients in the experimental group received Zalust or antagonist combination therapy; the included subjects had been evaluated for small airway abnormalities; and shortterm oral hormones or inhaled short-acting $\beta 2$ receptor agonists were administered in cases of rapid asthma deterioration.

The following exclusion criteria were applied: the subjects showed symptoms of, or had been recently diagnosed with and treated for respiratory tract infections; the patients had recently been administered leukotriene receptor antagonist drugs; the subjects suffered from other chronic respiratory diseases such as chronic obstructive pulmonary disease; the patients had a history of smoking 
for more than 10 years; the subjects or data overlapped with each other; and the patients were not treated.

Two senior experts were invited to independently screen the topics, abstracts, and full text articles. If there were inconsistencies between the two experts, a consensus conclusion was obtained through discussion, or a third expert was invited to arbitrate.

\section{Quality evaluation}

In this study, the Newcastle-Ottawa Scale (NOS) of the Cochrane system was used to evaluate the pathological control studies, and the star system (a maximum of 9 stars) was applied to evaluate the study objects, case comparisons, and group-to-group comparisons. The selected literature with 7 stars and above were considered high quality (low risk bias); while literature with 1 star or no stars were considered low quality (high risk bias); and studies with 2-6 stars were considered medium quality (medium risk bias). The funnel chart method was adopted to identify the positioning bias and publication bias of the literature. Taking the sample size (or the inverse of the standard error of the effect size) and the effect size (or the logarithm of the effect size) as scatter plots, and the funnel chart could be applied to directly observe the whether the effect size of the original study was related to the sample size. When there is publication bias, the funnel graph exhibited asymmetry, showing a skewed distribution. The sensitivity was analyzed based on the forest map. The studies that were relatively different from the $95 \%$ confidential interval (CI) distribution of the overall sample were excluded until the fixed effects model (FEM) could be used for analysis. The $\mathrm{I}^{2}$ statistic test method was used to evaluate the heterogeneity of the literature. For the $\mathrm{I}^{2}$ statistic test method, when $\mathrm{I}^{2}$ was 0 , it meant that each study was completely homogeneous, and if $\mathrm{I}^{2}>50 \%$, it meant that there was heterogeneity among the studies.

Two senior experts were invited to independently evaluate the quality of documents, and three preliminary experiments were required before screening. If there were inconsistencies between the two experts, a consensus conclusion could be obtained through discussion, or a third expert could be invited to arbitrate.

\section{Data extraction}

Two experts were invited to extract data independently with unified Excel table, and three preliminary experiments should be performed before extraction. If there were inconsistencies between the two experts, a consensus conclusion could be obtained through discussion, or a third expert could be invited to arbitrate. The data extracted and included in the study covered the first author and the year of publication, number of subjects, the duration and treatment time of the subjects' asthma, the grouping of subjects and the intervention treatment methods in the experimental group and the control group respectively, and evaluation index and parameters on effectiveness for the treatment of asthma, such as the one-second forced expiratory volume (FEV1), asthma control questionnaire (ACQ) score, adverse events, forced vital capacity (FVC), and peak expiratory flow (PEF).

\section{Statistical methods}

The Review manager 5.3 software was adopted for metaanalysis. Mean difference (MD) or standardized MD (SMD) and $95 \%$ CI were used for the continuous variables of the study data were selected for the efficacy analysis statistics for the continuous variables. A heterogeneity test ( $Q$ test) was performed on the included documents. The Review Manager software was used to assess the risk bias of the included studies. Each effect was expressed using a $95 \%$ CI. When $\mathrm{P}>0.1$ and $\mathrm{I}^{2}<50 \%$, the fixed-effects model was applied for meta-analysis. When $\mathrm{P}<0.1$ and $\mathrm{I}^{2}>50 \%$, the random effects model was used for meta-analysis.

\section{Results}

\section{Literature results overview}

From the 250 documents obtained, 140 articles were eliminated through abstracts and titles, 97 articles were eliminated after reading the full text. Finally, 13 studies were obtained for this meta-analysis. The excluded studies mainly included non-asthmatic patients or asthmatic patients with other types of respiratory diseases (48 articles), 19 articles related to animal experiments; 39 articles contained repeated subjects, 68 articles had unavailable research information, 51 articles referred to non-respiratory system or small airway parameters, and 17 articles lacked original research data. The basic information of the included literature is shown in Table 1. The included studies were published between 2012 and 2018, and the duration of asthma ranged from 2-12 weeks. Figure 1 shows the NOS rating. A total of 4 studies achieved 7 stars and 


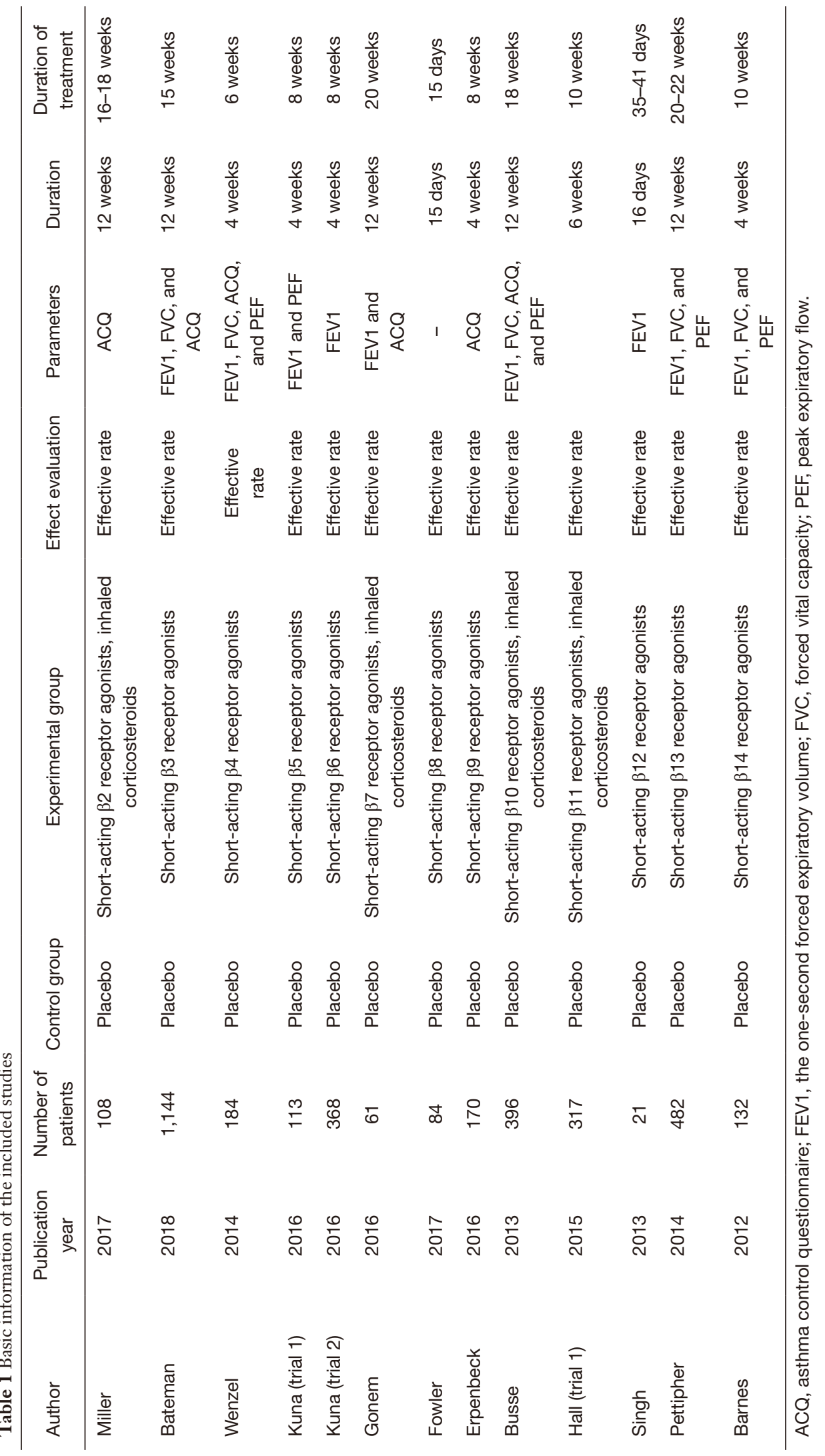


above, 9 studies achieved 2-6 stars, and none had 0-2 stars, suggested that all the included literature were of medium to high quality.

\section{Evaluating the risk bias of the included studies}

The Review Manager software was used to evaluate the risk bias of the included studies (Figures 2,3). The random sequence generation (selection bias), allocation hiding (selection bias), blind method of result evaluation (measurement bias), incomplete result data (follow-up bias), and selective reporting (reporting bias) showed low risk bias. The blinding methods (implementation bias) of the subjects and researchers and other biases were approximately $50 \%$. Except for the articles by Erpenbeck et al. [2016] (12) and Pettipher et al. [2014] (13), the risk bias of all included studies was obviously low.

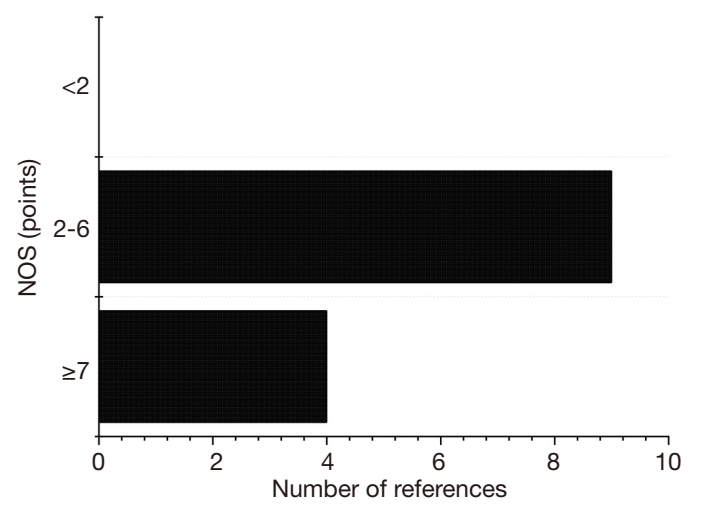

Figure 1 NOS score of literature.

\section{Incidences for adverse events of asthma}

The adverse events that occurred during and after treatment of asthma patients in the control group and the experimental group were analyzed (Figure 4). The most common adverse events were nasopharyngitis, headache, asthma, respiratory infections, and gastrointestinal diseases. Each type of adverse event was included in the metaanalysis. The report by Pettipher et al. (13) accounted for the highest percentage of the final combined results $(24.7 \%)$, followed by Hall et al. (trial 1) (22.1\%) and Erpenbeck et al. (12) (15.0\%). In addition, the horizontal line of the $95 \%$ CI of most studies were in the right of the invalid vertical line, and the horizontal line of the research crossed the invalid vertical line. In a few studies, the horizontal line of the $95 \%$ CI was in the left of the invalid vertical line. In the included 13 studies, 915 subjects were analyzed in the control group in total, and 543 subjects were researched in the experimental group in total. There was no statistical heterogeneity in the incidence of adverse events between the control group and the experimental group $\left(\mathrm{Chi}^{2}=7.97\right.$, $\mathrm{I}^{2}=12 \%$, and $\mathrm{P}=0.34$ ). The combined effect size (diamond block) was on the right side of the invalid line, the odds ratio (OR) value was 1.06 , and the $95 \%$ CI was $(0.84,1.33)$. The fixed-effects model analysis indicated that there was no statistically extreme difference in the occurrence of adverse events between the control group and the experimental group $(\mathrm{Z}=0.46, \mathrm{P}=0.64)$.

Figure 5 shows a funnel chart for the distribution of adverse events caused by asthma in the control group and the experimental group. It revealed that the circles included in the study were concentrated in the top area (showing

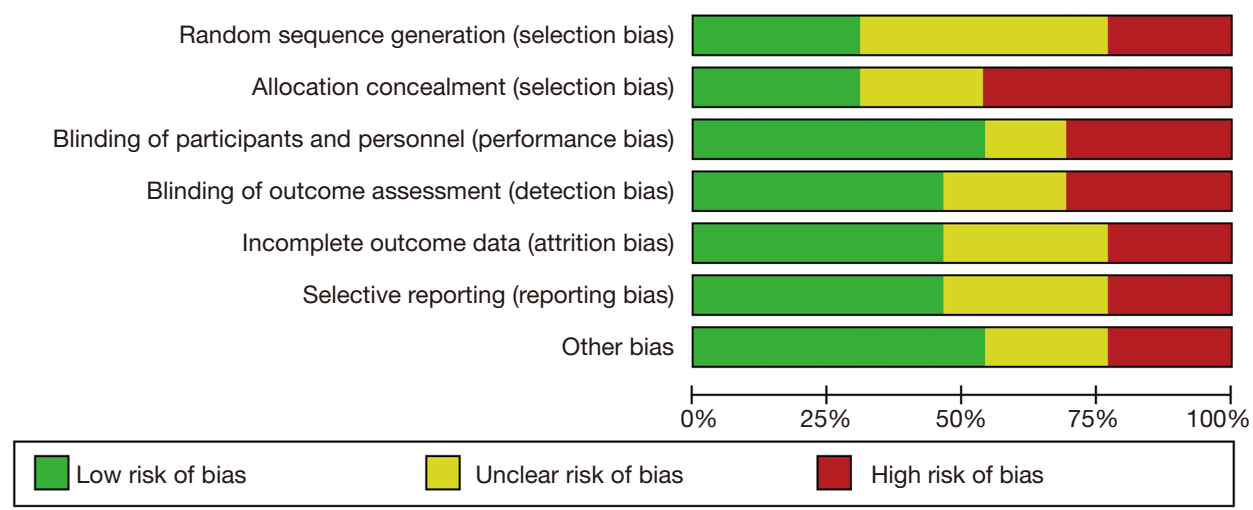

Figure 2 The risk bias analysis of the included studies. 


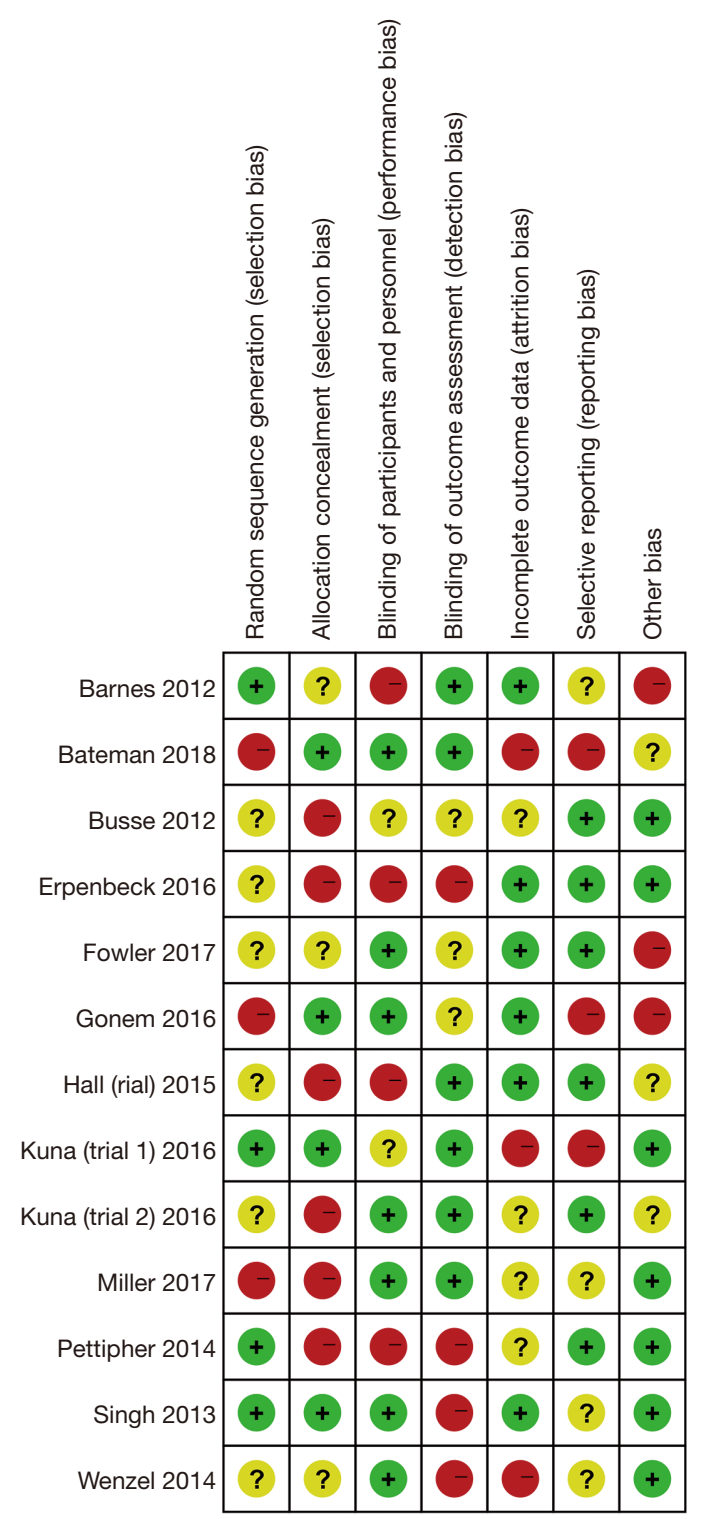

Figure 3 Evaluation of multiple risk bias with multiple studies. Note: “+” meant "low risk of bias”; "?” referred to "unclear risk of bias”; and “_” referred to "high risk of bias".

high accuracy), and they were distributed on both sides of the midline, in a roughly symmetrical form. Therefore, there was no publication bias in the included literature.

\section{Comparison of FEV1 of patients in two groups}

The effects of different treatment methods on FEV1 during the treatment were compared in the control group and the experimental group, and the results were illustrated in Figure 6. The research results of Pettipher et al. [2014] (13) accounted for the highest percentage $(43.3 \%)$ of the final combined results, followed by the research results of Wenzel et al. (14) (22.5\%), and Barnes et al. (15) (16.2\%). In addition, the horizontal lines of the $95 \%$ CI of most studies were in the left of the invalid vertical line, only a few studies had no horizontal line crossed with the invalid vertical line, and the horizontal lines of the $95 \%$ CI of a few studies were in the right 


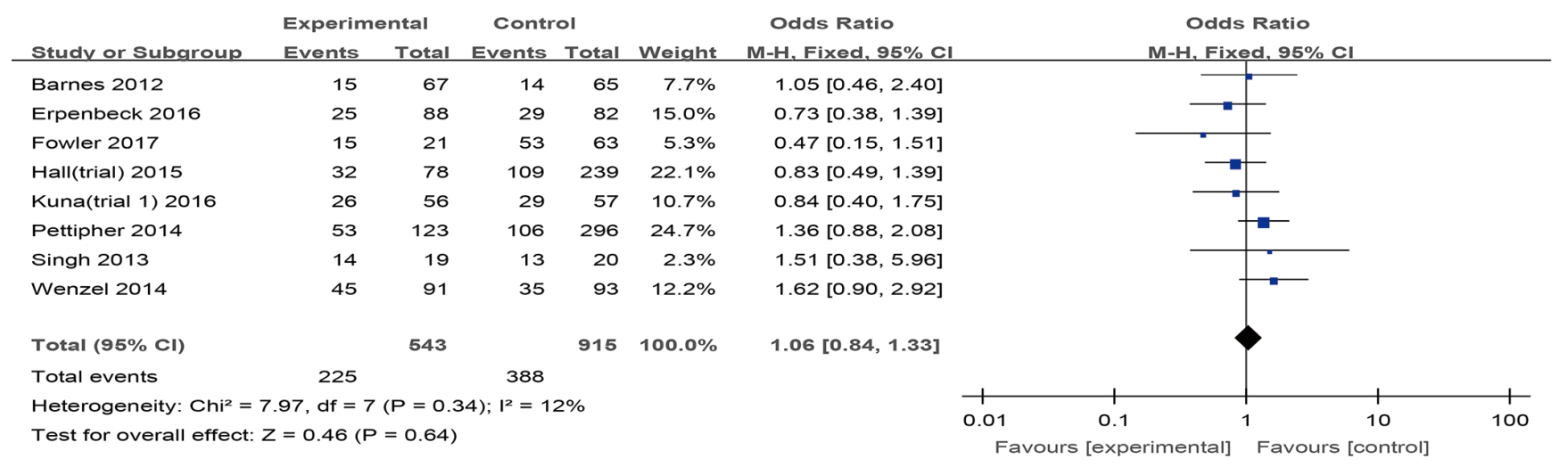

Figure 4 Incidence for adverse events of asthma. M-H, Mantel-Haenszel; CI, confidence interval.

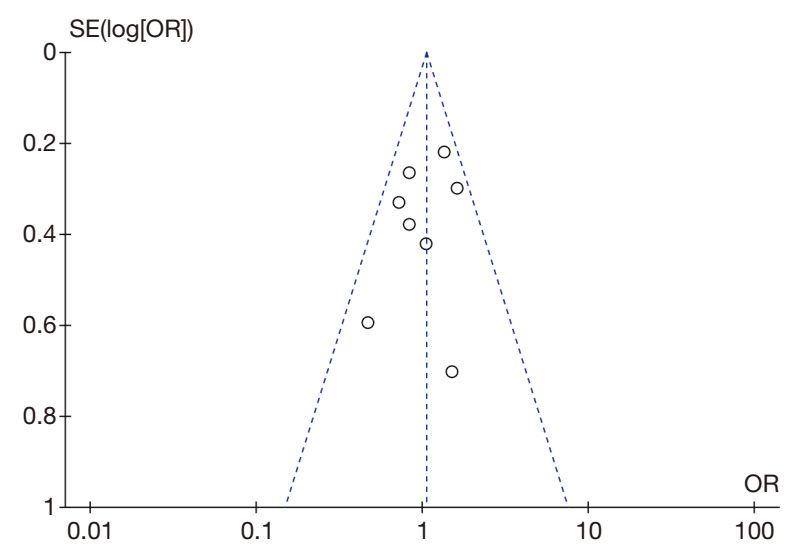

Figure 5 Funnel map for adverse events distribution of asthma. SE, standard error; OR, odds ratio.

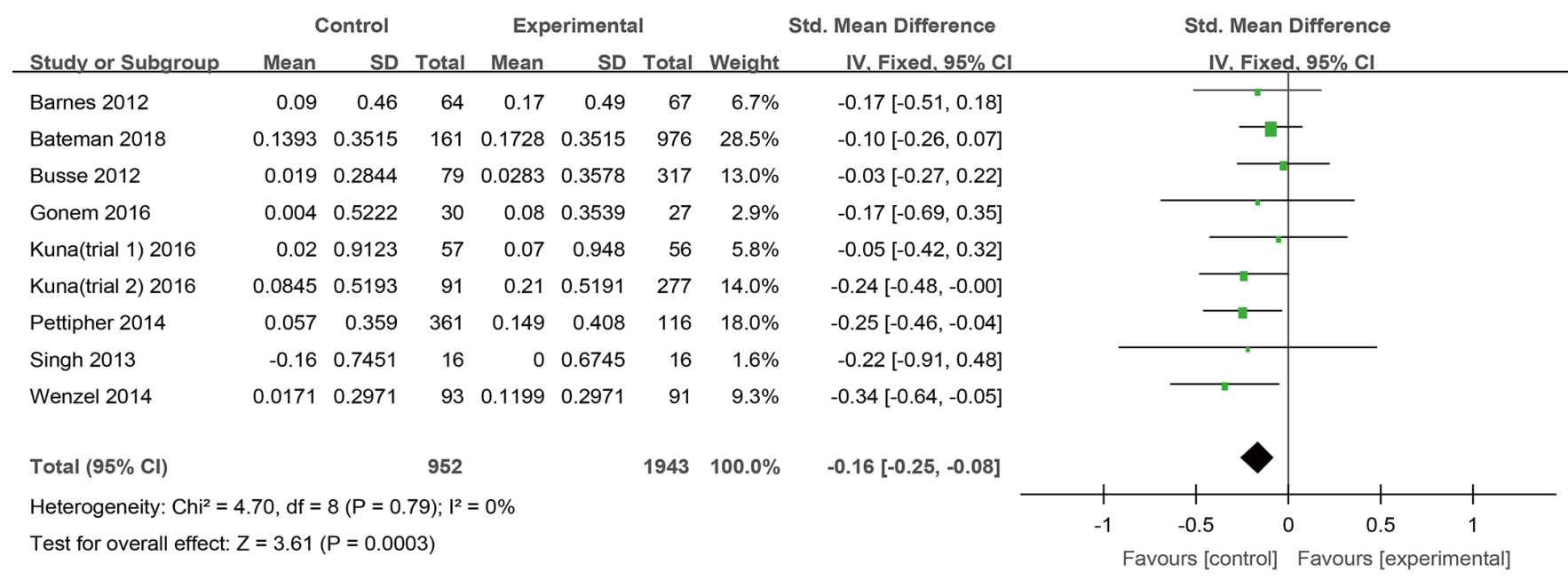

Figure 6 Comparison of FEV1. SD, standard deviation; CI, confidence interval. 
side the invalid vertical line. Among the 13 included documents, there were 591 subjects in the control group in total and 346 subjects in the experimental group in total. The treatment methods of the control group and the experimental group showed no statistical heterogeneous in the effect on FEV1 $\left(\mathrm{Chi}^{2}=4.70, \mathrm{I}^{2}=0 \%\right.$, and $\left.\mathrm{P}=0.79\right)$. The combined effect size (diamond block) was on the left side of the invalid line, the OR value was -0.16 , and the $95 \%$ CI was $(-0.25,-0.08)$. The fixed-effects model analysis indicated that the FEV1 in the control group was obviously lower than in contrast to the experimental group $(\mathrm{Z}=3.61, \mathrm{P}=0.0003)$.

The funnel chart in Figure 7 shows the effects of treatment or placebo on FEV1 in the control group and the experimental group, respectively. It revealed that the circles included in the study were concentrated in the top area (showing high accuracy), and they were distributed on both sides of the midline, in a roughly symmetrical form.

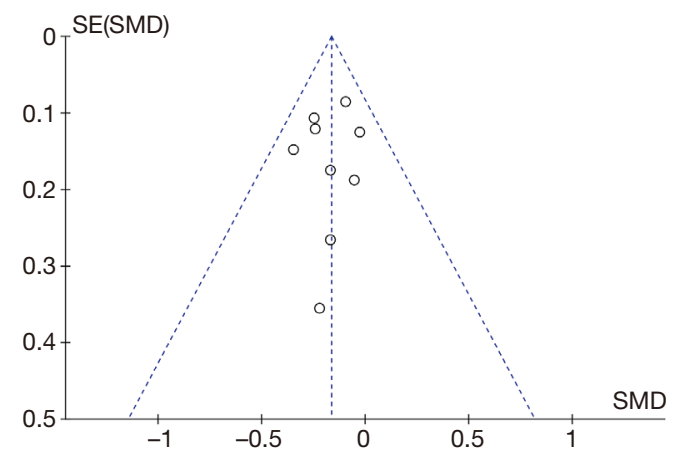

Figure 7 Funnel map for change distribution of FEV1. Note: SE (SMD) referred to standard error, and SMD referred to effect size.
Therefore, there was no publication bias in the included literature.

\section{Comparison on ACQ score}

The ACQ scores for patients in the two groups were compared (Figure 8). The research results of Miller et al. [2017] (16) accounted for the highest percentage (29.7\%) of the final combined results, followed by the research results of Bateman et al. (17) (22.7\%), and Erpenbeck et al. (12). $(17.5 \%)$. In addition, the horizontal lines of the $95 \%$ CI of most studies were in the left of the invalid vertical line, only a few studies had no horizontal line crossed with the invalid vertical line, and the horizontal lines of the $95 \%$ CI of a few studies were in the right side the invalid vertical line. Among the 13 included documents, there were 546 subjects in the control group in total and 1,686 subjects in the experimental group in total. The ACQ scores of the experimental group and the control group showed no statistical heterogeneous $\left(\mathrm{Chi}^{2}=8.58, \mathrm{I}^{2}=42 \%\right.$, and $\left.\mathrm{P}=0.13\right)$. The combined effect size (diamond block) was on the left side of the invalid line, the OR value was -0.12 , and the $95 \%$ CI was $(-0.21,-0.03)$. The fixed-effects model analysis indicated that ACQ score in the control group was obviously lower than in contrast to the experimental group $(\mathrm{Z}=2.60, \mathrm{P}=0.009)$.

Figure 9 shows a funnel chart comparing the ACQ scores for patients in the control group and the experimental group. It revealed that the circles included in the study were distributed on both sides of the midline, but they were not symmetrical. Therefore, there was publication bias in the included studies.

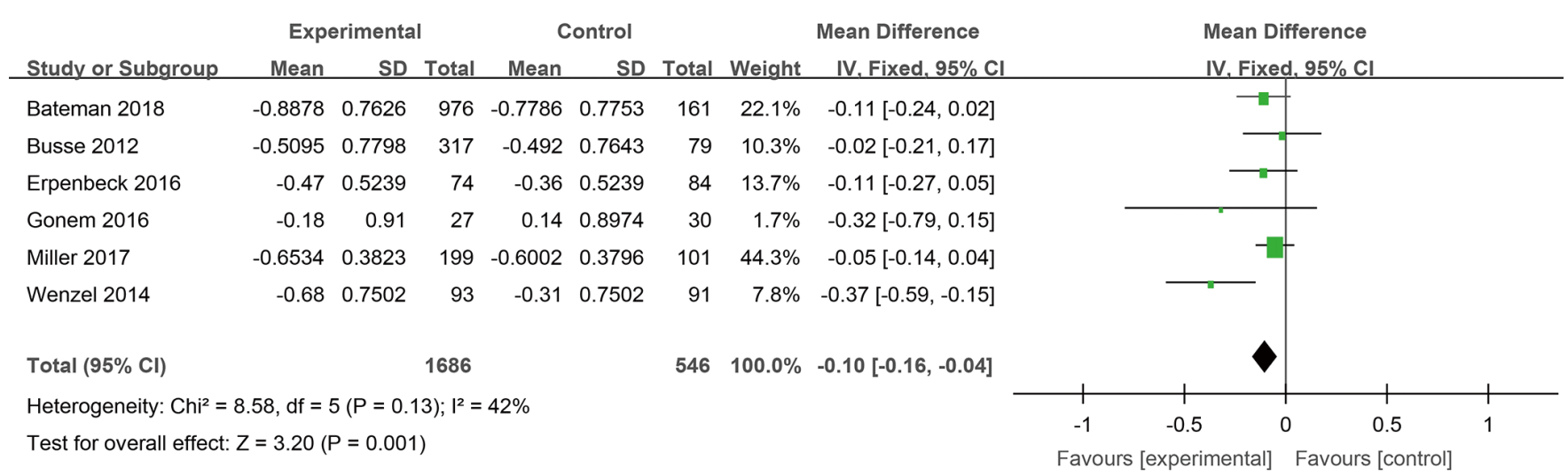

Figure 8 Comparison on ACQ score of patients. SD, standard deviation; CI, confidence interval. 


\section{A comparison of the PEF in asthma patients}

Figure 10 shows the PEF results for patients in the two groups. The research results of Pettipher et al. [2014] (13) accounted for the highest percentage $(31.9 \%)$ of the final combined results, followed by the research results of Wenzal et al. (14). (23.0\%), and Busse et al. (18) (22.9\%). In addition, the horizontal lines of the $95 \%$ CI of most studies were on the left of the invalid vertical line, and only a few studies had no horizontal line crossed with the invalid vertical line. The horizontal lines of the $95 \%$ CI of a few studies were on the right side the invalid vertical line. Among the 13 included documents, there were a total of 397 subjects in the control group and 1,116 subjects in the experimental group. The PEF results of the experimental group and the control group showed statistical heterogeneity $\left(\mathrm{Chi}^{2}=7.87, \mathrm{I}^{2}=49 \%\right.$, and $\left.\mathrm{P}=0.10\right)$. The combined effect size (diamond block) was on the left side of the invalid line, the OR value was -0.04 , and the $95 \%$ CI was -0.16 to 0.08 . The fixed-effects model analysis

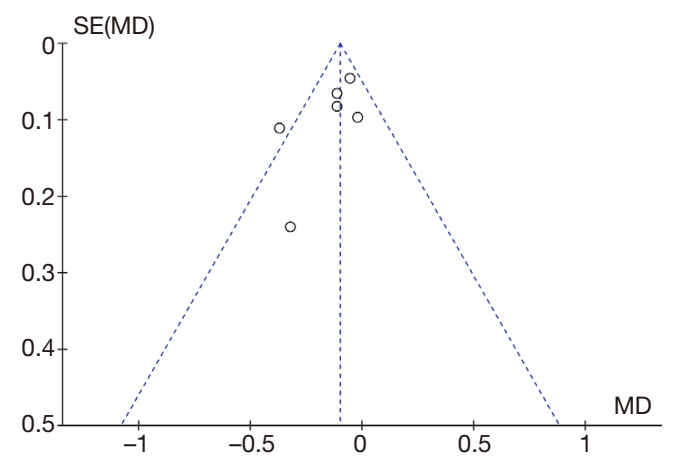

Figure 9 Funnel map for distribution of ACQ score. Note: SE (SMD) referred to standard error, and SMD referred to effect size. indicated that the PEF results in the control group was not significantly different to that observed in the experimental group $(\mathrm{Z}=0.64, \mathrm{P}=0.052)$.

Figure 11 shows a funnel chart comparing the PEF in patients in the control group and the experimental group. It revealed that the circles included in the study were distributed on both sides of the midline, but they were not symmetrical. Therefore, there was publication bias in the included studies.

\section{Astbma exacerbation}

The asthma exacerbation experienced by patients in the two groups was compared, and the results are illustrated in Figure 12. The research results of Pettipher et al. [2014] (13) accounted for the highest percentage (31.7\%) of the final combined results, followed by the research results of Busse et al. (18) (21.3\%), and Wenzel et al. (14) (21.0\%). In addition, the horizontal lines of the $95 \%$ CI of most studies were on the left of the invalid vertical line, and only a few studies had no horizontal line crossed with the invalid vertical line. The horizontal lines of the $95 \%$ CI of a few studies were on the right side of the invalid vertical line. Among the 13 included documents, there were a total of 515 subjects in the control group and 1,816 subjects in the experimental group. The asthma exacerbation of the experimental group and the control group showed no statistical heterogeneity $\left(\mathrm{Chi}^{2}=3.72, \mathrm{I}^{2}=0 \%\right.$, and $\left.\mathrm{P}=0.45\right)$. The combined effect size (diamond block) was on the left side of the invalid line, the OR value was 0.69 , and the $95 \%$ CI was 0.44 to 1.09 . The fixed-effects model analysis indicated that asthma exacerbation in the control group was not significantly different to that observed in the experimental group $(\mathrm{Z}=1.57, \mathrm{P}=0.12)$.

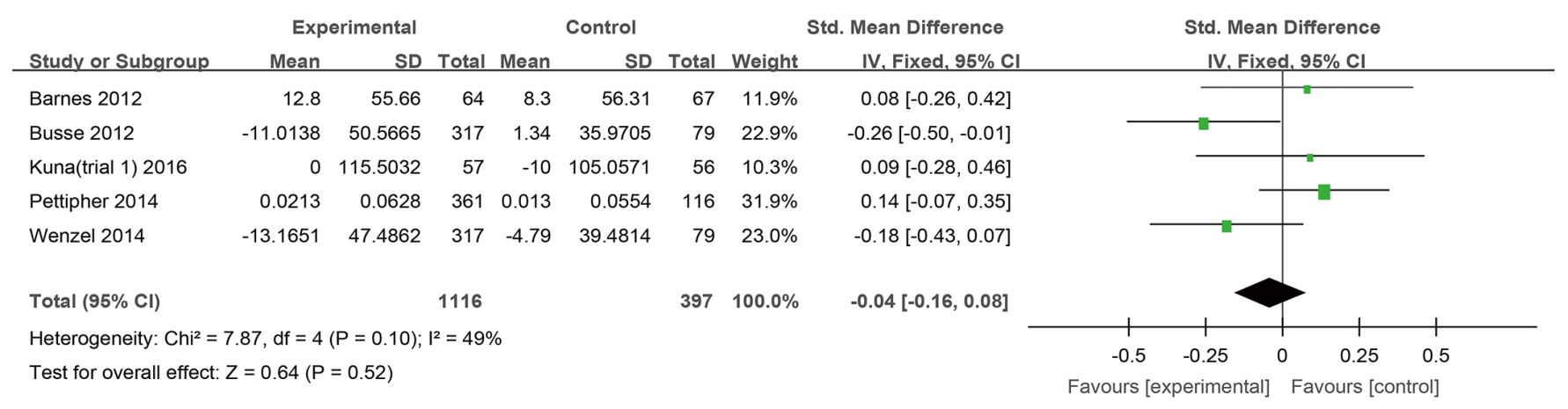

Figure 10 Comparison on EPF of patients. SD, standard deviation; CI, confidence interval. 
Figure 13 shows a funnel chart comparing asthma exacerbation in patients in the control group and the experimental group. It revealed that the circles included in the study were concentrated in the top area (showing high accuracy), and they were distributed on both sides of the midline, in a roughly symmetrical form. Therefore, there was no publication bias in the included literature.

\section{Discussion}

Of the 13 articles included, 12 adopted the randomized controlled grouping method, and only 1 adopted the retrospective analysis method, which resulted in bias in the study. However, this had little effect on the results of the meta-analysis in general. The research of a single sample may be unstable. The meta-analysis was applied to quantitatively synthesize each included study. This not only

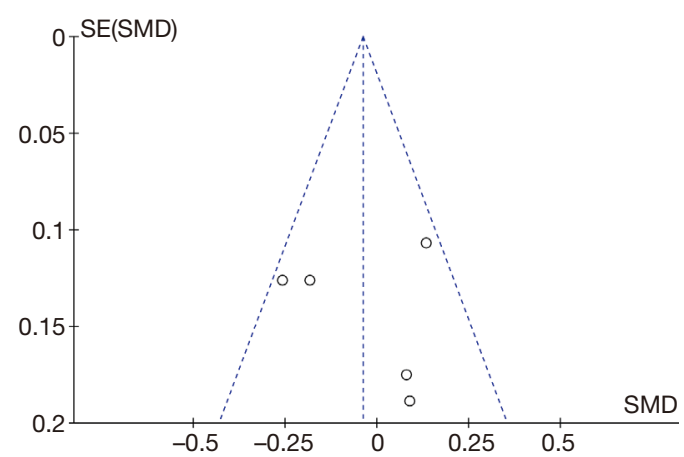

Figure 11 Funnel map for change distribution of PEF. Note: SE (SMD) referred to standard error, and SMD referred to effect size. avoids the differences among the studies caused by sampling from different populations, but also assigns different weights to the results according to the sample size of each study, thereby increasing the sample size and improving the credibility of the conclusions (19). The quality of any meta-analysis mainly depends on the authenticity and completeness of the analyzed literature. Due to the objective influence of the included studies, the number of included reports in this meta-analysis was limited. Future investigations should use a larger sample size to prevent bias.

This meta-analysis explored the efficacy of competitive antagonists in the treatment of asthma. There was no statistically heterogeneity on FEV1 between the different treatment methods of the control group and the experimental group $\left(\mathrm{Chi}^{2}=4.70, \mathrm{I}^{2}=0 \%\right.$, and $\left.\mathrm{P}=0.79\right)$, and the FEV1 of the control group was much lower than that of the experimental group $(\mathrm{Z}=3.61, \mathrm{P}=0.0003)$. It indicated that compared with placebo, competitive antagonists could improve the FEV1 obviously before bronchodilator. There was no statistical heterogeneity in the occurrence of adverse events between the control group and the experimental group $\left(\mathrm{Chi}^{2}=7.97, \mathrm{I}^{2}=12 \%\right.$, and $\left.\mathrm{P}=0.34\right)$, and the difference in the occurrence of adverse events between the two groups was not significantly different from each other $(\mathrm{Z}=0.46$, $\mathrm{P}=0.64)$. These results indicated that the occurrence of adverse events was not affected by asthma competitive antagonists. There was no statistical heterogeneity in the ACQ scores of the two groups of patients $\left(\mathrm{Chi}^{2}=8.58\right.$, $\mathrm{I}^{2}=42 \%$, and $\left.\mathrm{P}=0.13\right)$. The ACQ scores of the control group were observably lower than those of the experimental group

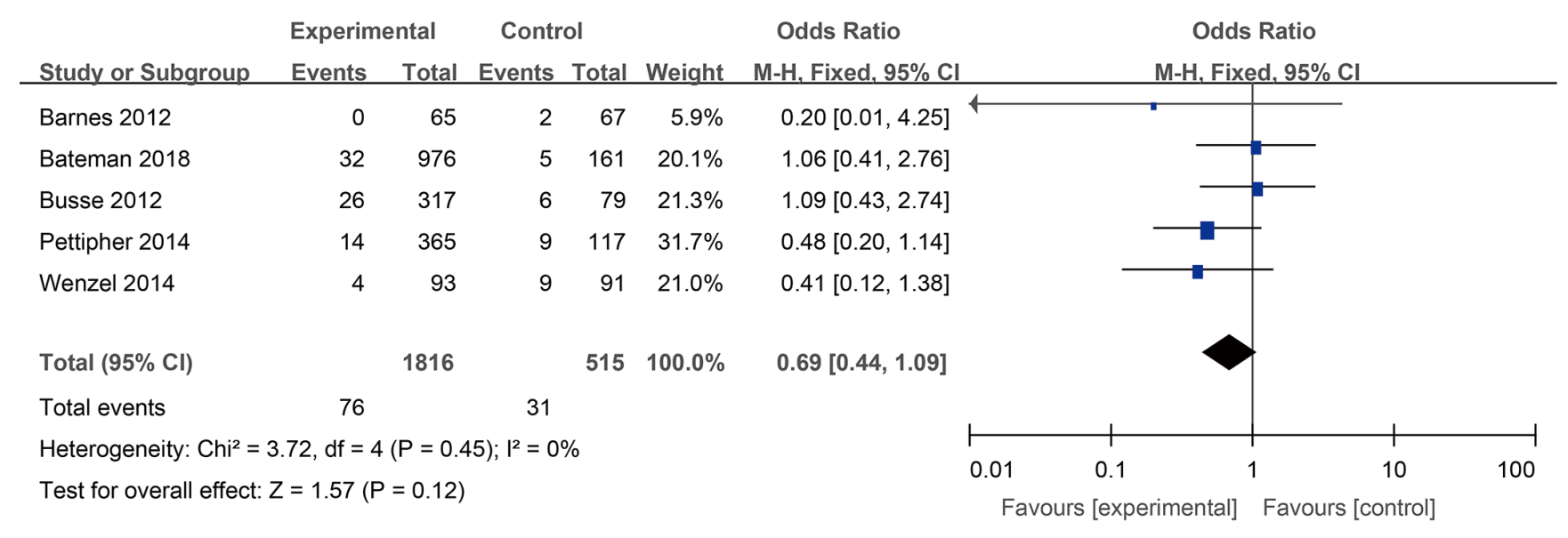

Figure 12 Comparison on asthma exacerbation. M-H, Mantel-Haenszel; CI, confidence interval. 


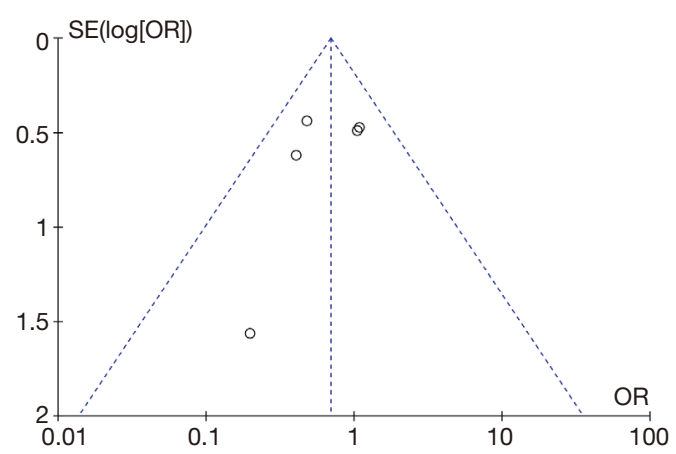

Figure 13 Funnel map for incidence of asthma exacerbation. SE, standard error; OR, odds ratio.

$(\mathrm{Z}=2.60, \mathrm{P}=0.009)$, indicating that competitive antagonists of asthma reduced the patient's ACQ score visibly. This was consistent with the results of Kuna et al. [2016] (20) showing that competitive antagonists of CRTH2 effectively improved FEV1 and lowered ACQ scores in patients with asthma, and thus the competitive antagonists were effective in treating asthma.

\section{Conclusions}

In this study, a meta-analysis was performed for documents on competitive antagonists for treatment of asthma taking placebo as a control, aiming to explore the effectiveness of competitive antagonists in the treatment of asthma. The results showed that competitive antagonists of CRTH2 could effectively improve FEV1 and lower ACQ scores in patients with asthma, and competitive antagonists were effective in treating asthma. However, the meta-analysis in this study also showed limitations due to the influence of various confounding factors. The documents selected were case-control, so there was a survival bias; there were many predisposing factors for asthma, and many risk factors and indicators may not be included in the study, so that the combined effect size was greatly reduced. It will take some follow-up analysis of patients with asthma in future to explore the effectiveness of antagonists in treating asthma, so as to improve the results of meta-analysis. In short, this study provided theoretical basis and data support for clinical treatment of asthma.

\section{Acknowledgments}

Funding: None.

\section{Footnote}

Reporting Checklist: The authors have completed the PRISMA reporting checklist. Available at http://dx.doi. org/10.21037/apm-21-412

Conflicts of Interest: All authors have completed the ICMJE uniform disclosure form (available at http://dx.doi. org/10.21037/apm-21-412). The authors have no conflicts of interest to declare.

Ethical Statement: The authors are accountable for all aspects of the work in ensuring that questions related to the accuracy or integrity of any part of the work are appropriately investigated and resolved.

Open Access Statement: This is an Open Access article distributed in accordance with the Creative Commons Attribution-NonCommercial-NoDerivs 4.0 International License (CC BY-NC-ND 4.0), which permits the noncommercial replication and distribution of the article with the strict proviso that no changes or edits are made and the original work is properly cited (including links to both the formal publication through the relevant DOI and the license). See: https://creativecommons.org/licenses/by-ncnd/4.0/.

\section{References}

1. Rivero A, Liang J. Anti-IgE and anti-IL5 biologic therapy in the treatment of nasal polyposis: a systematic review and Meta-analysis. Ann Otol Rhinol Laryngol 2017;126:739-47.

2. Kallur L, Gonzalez-Estrada A, Eidelman F, et al. Pharmacokinetic drug evaluation of mepolizumab for the treatment of severe asthma associated with persistent eosinophilic inflammation in adults. Expert Opin Drug Metab Toxicol 2017;13:1275-80.

3. Mahemuti G, Zhang H, Li J, et al. Efficacy and side effects of intravenous theophylline in acute asthma: a systematic review and meta-analysis. Drug Des Devel Ther 2018;12:99-120.

4. Iftikhar IH, Schimmel M, Bender W, et al. Comparative efficacy of anti-IL-4, IL-5 and IL-13 drugs for treatment of eosinophilic asthma: a network meta-analysis. Lung 2018;196:517-30.

5. Zhang HP, Jia CE, Lv Y, et al. Montelukast for prevention and treatment of asthma exacerbations in adults: 
systematic review and meta-analysis. Allergy Asthma Proc 2014;35:278-87.

6. Singh D, Cadden P, Hunter M, et al. Inhibition of the asthmatic allergen challenge response by the CRTH2 antagonist OC000459. Eur Respir J 2013;41:46-52.

7. Sobieraj DM, Baker WL, Nguyen E, et al. Association of inhaled corticosteroids and long-acting muscarinic antagonists with asthma control in patients with uncontrolled, persistent asthma: a systematic review and meta-analysis. JAMA 2018;319:1473-84.

8. Hall IP, Fowler AV, Gupta A, et al. Efficacy of BI 671800, an oral CRTH2 antagonist, in poorly controlled asthma as sole controller and in the presence of inhaled corticosteroid treatment. Pulm Pharmacol Ther 2015;32:37-44.

9. Wang FP, Liu T, Lan Z, et al. Efficacy and safety of anti-interleukin-5 therapy in patients with asthma: a systematic review and meta-analysis. PLoS One 2016;11:e0166833.

10. Fowler A, Koenen R, Hilbert J, et al. Safety, tolerability, pharmacokinetics, and pharmacodynamics of the novel CRTH2 antagonist BI 1021958 at single oral doses in healthy men and multiple oral doses in men and women with well-controlled asthma. J Clin Pharmacol 2017;57:1444-53.

11. Castro-Rodriguez JA, Rodriguez-Martinez CE, Ducharme FM. Daily inhaled corticosteroids or montelukast for preschoolers with asthma or recurrent wheezing: a systematic review. Pediatr Pulmonol 2018;53:1670-7.

12. Erpenbeck VJ, Popov TA, Miller D, et al. The oral CRTh2 antagonist QAW039 (fevipiprant): a phase II study in uncontrolled allergic asthma. Pulm Pharmacol Ther 2016;39:54-63.

13. Pettipher R, Hunter MG, Perkins CM, et al. Heightened

Cite this article as: Lei Z, Luo Q, Yang W, Su Q, Zuo Y. Meta-analysis of competitive antagonists in asthma treatment. Ann Palliat Med 2021;10(3):3235-3246. doi: 10.21037/apm-21-412 response of eosinophilic asthmatic patients to the CRTH2 antagonist OC000459. Allergy 2014;69:1223-32.

14. Wenzel SE, Hopkins R, Saunders M, et al. Safety and efficacy of ARRY-502, a potent, selective, Oral CRTh2 antagonist, in patients with mild to moderate Th2-driven asthma. J Allergy Clin Immunol 2014;133:AB4.

15. Barnes N, Pavord I, Chuchalin A, et al. A randomized, double-blind, placebo-controlled study of the CRTH2 antagonist OC000459 in moderate persistent asthma. Clin Exp Allergy 2012;42:38-48.

16. Miller D, Wood C, Bateman E, et al. A randomized study of BI 671800, a CRTH2 antagonist, as add-on therapy in poorly controlled asthma. Allergy Asthma Proc 2017;38:157-64.

17. Bateman ED, O'Brien C, Rugman P, et al. Efficacy and safety of the CRTh2 antagonist AZD1981 as add-on therapy to inhaled corticosteroids and long-acting beta2agonists in patients with atopic asthma. Drug Des Devel Ther 2018;12:1093-106.

18. Busse WW, Wenzel SE, Meltzer EO et al. Safety and efficacy of the prostaglandin D2 receptor antagonist AMG 853 in asthmatic patients. J Allergy Clin Immunol 2013;131:339-45.

19. Gonem S, Berair R, Singapuri A, et al. Fevipiprant, a prostaglandin D-2 receptor 2 antagonist, in patients with persistent eosinophilic asthma: a single-centre, randomised, double-blind, parallel-group, placebocontrolled trial. Lancet Respir Med 2016;4:699-707.

20. Kuna P, Bjermer L, Tornling G. Two phase II randomized trials on the CRTh2 antagonist AZD1981 in adults with asthma. Drug Des Devel Ther 2016;10:2759-70.

(English Language Editor: J. Teoh) 